\title{
Consensus-based recommendations in respiratory medicine
}

\author{
Kevin C. Wilson \\ Affiliation: Dept of Medicine, Boston University School of Medicine, Boston, MA, USA. \\ Correspondence: Kevin C. Wilson, ATS Documents, 10 Whitney Ave, Westwood, MA 02090, USA. \\ E-mail: kwilsondthoracic.org
}

@ERSpublications

When conducted by experts in a field, a Delphi-like process called the CORE process can yield recommendations that are highly concordant with guideline recommendations that have been informed by a systematic review of the literature https://bit.ly/3klCSua

Cite this article as: Wilson KC. Consensus-based recommendations in respiratory medicine. Eur Respir J 2020; 56: 2002889 [https://doi.org/10.1183/13993003.02889-2020].

\section{Introduction}

5 years ago, I watched one of the world's most prominent guideline methodologists present a flawless systematic review to inform a guideline committee's recommendations. At the conclusion of the presentation, a member of the guideline committee turned to me, smiled, and whispered, "that was great work, but I could have told you the results 9 months ago." The comment stuck with me.

2 years later a unique opportunity emerged. The American Thoracic Society (ATS) had eight clinical practice guidelines in press. All of the guidelines had been developed using Institute of Medicine-adherent guideline methodology [1], which included a full systematic review of the literature with meta-analyses and grading to inform each recommendation. The guidelines were not yet published and had never been publicly presented. With the help of some colleagues, I recruited experts and asked them to answer the same questions as the guidelines using a Delphi-like process that my research fellow subsequently termed the Convergence of Opinion on Recommendations and Evidence (CORE) process.

I expected a substantial number of the recommendations to differ, which would provide the evidence that I needed to preach to guideline developers about the need to follow standards of guideline development or risk making incorrect recommendations. However, the findings were different than I expected. Ninety-eight percent of the CORE-derived recommendations were concordant with the guideline recommendations when a threshold of $70 \%$ agreement among the experts was necessary to make a recommendation [2]. While our study received praise [3], it also received criticism [4-6], ironically using many of the same arguments and anecdotes that I had used over the previous decade when teaching about guideline methodology. Notably, none of the criticism doubted the findings but, rather, expressed concern that the CORE process was a slippery slope that would be overused and, if used indiscriminately, could lead to incorrect recommendations.

To ensure that the findings were trustworthy, we repeated our study using guidelines on idiopathic pulmonary fibrosis [7] and community-acquired pneumonia (undergoing peer review) as comparators, and again found that the CORE process yielded recommendations that were highly concordant with recommendations informed by a systematic review of the literature. The CORE process has transitioned from being validated to being utilised $[8,9]$. This edition of the European Respiratory Journal includes one of the first guidance documents developed with the CORE process, in which experts formulate suggestions about pulmonary rehabilitation during the COVID-19 pandemic [10]. 


\section{What is the CORE process?}

The CORE process is a modified Delphi process. It begins by formulating clinical questions using the population, intervention, comparator, and outcomes (PICO) format. Those questions are then discussed by the participants to ensure that the questions accurately convey their intent, are clearly written, and all terms have been defined. Once there is agreement on the precise questions, an electronic survey is conducted; for each question, the respondent must choose among a strong or weak/conditional recommendation for or against the course of action or no recommendation, and also has the opportunity to provide written comments. A second survey is issued that is identical to the first, except the numerical responses and comments from the first survey are provided. Upon completion of the second survey, course of actions that garner more than $70 \%$ agreement are adopted as recommendations. Courses of action for which there is less than $70 \%$ agreement should not yield a clinical recommendation until informed by a systematic review of the literature. Using a higher agreement threshold to yield a recommendation is permissible; both the total number of recommendations and the number of discordant recommendations will decrease. Finally, the recommendations are discussed among the participants to ensure that they reflect the intent of the participants. Recommendations generated using the CORE process are not graded.

\section{How should the CORE process be used?}

The ideal context in which to use the CORE process is still being determined. It was initially investigated as a potential screening tool to determine which guideline questions require a full systematic review of the literature; it was never intended to be an alternative to systematic review-informed recommendations when uncertainty exists. It has been suggested that the CORE process may be appropriate to use when there is an expectation that no or only very low quality empirical evidence exists, such as the early stages of a pandemic, since a systematic review is unlikely to identify important empirical evidence that is unknown to the participants. It has also been suggested that the CORE process may be appropriate to create recommendations when resources are limited, since the process is efficient and inexpensive, or as a placeholder to fill gaps until clinical practice guidelines informed by systematic reviews can be developed.

A particularly intriguing suggestion is that the CORE process may facilitate guideline implementation. It is well known that guideline recommendations frequently are not utilised by clinicians. There are many reasons but, among them, guideline recommendations tend to be written for single conditions, leaving clinicians with uncertainty regarding the applicability of a guideline recommendation to their patient with multiple morbidities [11]. The CORE process may be helpful in refining such recommendations, improving their usability. As an example, consider the US Preventative Services Task Force recommendation for lung cancer screening [12]. It states that current smokers or smokers who have quit in the past 15 years, are 55 to 80 years old, and have 30 pack-year tobacco history should undergo low-dose CT screening for lung cancer until they have not smoked for 15 years, develop a condition that limits life expectancy, or become unable or unwilling to undergo curative lung surgery. One problem with this recommendation is that there is no agreement on what constitutes a condition that limits life expectancy and therefore warrants foregoing lung cancer screening. Many smokers also have COPD, but at what point (i.e. lung function, oxygen saturation, etc.) does the condition become severe enough to warrant not doing lung cancer screening? The CORE process could be used to establish standard of care in such situations, essentially operating "within the guardrails" of the more rigorously developed guideline recommendation.

\section{Can the CORE process improve clinical outcomes?}

The impact of CORE-derived recommendations on clinical outcomes has never been studied. However, there is evidence that other consensus-based processes improve clinical outcomes, probably by standardising care and eliminating outlying dangerous practices. Prior to the Institute of Medicine Standards for Trustworthy Guidelines [1], guideline recommendations were usually derived using consensus by discussion. Numerous studies have shown that such guidelines improve clinical outcomes, such as the effect of guideline-adherent antibiotic regimens on mortality [13]. Similarly, checklists and policies/protocols are widely accepted as improving process-based and clinical outcomes, and most are the products of expert consensus.

\section{Why does the CORE process work?}

Agreement of CORE-derived recommendations with systematic review-informed recommendations probably reflects the evolving expectations of an expert. Whereas in the past experts routinely made recommendations based upon biological rationale and clinical experience with little regard for empiric evidence, experts have become defined by their knowledge of the evidence and their role in evidence generation. As a result, systematic reviews are increasingly unlikely to uncover important studies that are 
unknown to an expert panel. However, this highlights a key requirement for the success of the CORE process; the participants must be well-informed experts in the topic being addressed.

\section{What are the next steps?}

We welcome feedback, both positive and negative, pertaining recommendations created by the CORE process. We are particularly interested in input regarding the appropriate role of the CORE process and, just as importantly, situations in which the CORE process should never be used. We look forward to further using and refining the CORE process, as we are continually learning about the strengths and weaknesses of consensus in medicine.

Conflict of interest: K.C. Wilson developed, refined and validated the CORE process while employed as Chief of Guidelines and Documents for the American Thoracic Society.

\section{References}

1 IOM (Institute of Medicine). Clinical Practice Guidelines We Can Trust. Washington, The National Academies Press, 2011.

2 Schoenberg NC, Barker AF, Bernardo J, et al. A Comparative analysis of pulmonary and critical care medicine guideline development methodologies. Am J Respir Crit Care Med 2017; 196: 621-627.

Morice AH, Hart S, Millqvist E. Developing clinical guidelines. Am J Respir Crit Care Med 2018; 197: 837.

4 Shah R, Morgan RL, Falck-Ytter Y, et al. Have we not learned from past mistakes? Am J Respir Crit Care Med 2018; 197: 1499-1500.

5 Schünemann HJ, Brożek JL. No room for error in medicine - a case of déjà vu. Am J Respir Crit Care Med 2018; 197: 1501-1502.

6 Dahm P, Sultan S, Murad MH. A blast from the past - back to the 1970s. Am J Respir Crit Care Med 2018; 197: $1500-1501$.

7 Wilson KC, Schoenberg NC, Raghu G. Idiopathic pulmonary fibrosis guideline recommendations. need for adherence to institute of medicine methodology? Ann Am Thorac Soc 2019; 16: 681-686.

8 Wilson KC, Chotirmall SH, Bai C, et al. COVID-19: Interim Guidance on Management Pending Empirical Evidence. From an American Thoracic Society-led International Task Force. www.thoracic.org/covid/ covid-19-guidance.pdf Date last accessed: July 20, 2020.

9 Janssen DJA, Ekström M, Currow DC, et al. COVID-19: guidance on palliative care from a European Respiratory Society international task force. Eur Respir J 2020; 56: 2002583.

10 Spruit MA, Holland AE, Singh SJ, et al. COVID-19: interim guidance on rehabilitation in the hospital and post-hospital phase from a European Respiratory Society- and American Thoracic Society-coordinated international task force. Eur Respir J 2020; 56: 2002197.

11 Wilson KC, Gould MK, Krishnan JA, et al. An Official American Thoracic Society Workshop Report. A framework for addressing multimorbidity in clinical practice guidelines for pulmonary disease, critical illness, and sleep disorders. Ann Am Thorac Soc 2016; 13: S12-S21.

12 Moyer VA, US Preventive Services Task Force. Screening for lung cancer: US Preventive Services Task Force recommendation statement. Ann Intern Med 2014; 160: 330-338.

13 Wilson KC, Schünemann HJ. An appraisal of the evidence underlying performance measures for community-acquired pneumonia. Am J Respir Crit Care Med 2011; 183: 1454-1462. 